\title{
Corticosteroid-binding globulin contributes to the neuroendocrine phenotype of mice selected for extremes in stress reactivity
}

\author{
Gabriele E Mattos, Jan-Michael Heinzmann, Stefanie Norkowski, \\ Jean-Christophe Helbling ${ }^{1,2}$, Amandine M Minni, ${ }^{1,2}$, Marie-Pierre Moisan ${ }^{1,2}$ \\ and Chadi Touma
}

Max Planck Institute of Psychiatry, Research Group of Psychoneuroendocrinology, Kraepelinstrasse 2-10, 80804 Munich, Germany

${ }^{1}$ Institut National de la Recherche Agronomique (INRA), Laboratory of Nutrition and Integrative Neurobiology, UMR 1286, 146 Rue Leo Saignat, 33076 Bordeaux, France

${ }^{2}$ University of Bordeaux, Laboratory of Nutrition and Integrative Neurobiology, UMR 1286, 146 Rue Leo Saignat, 33076 Bordeaux, France
Correspondence should be addressed to G E Mattos Email mattos@mpipsykl.mpg.de or touma@mpipsykl.mpg.de

\begin{abstract}
Increasing evidence indicates an important role of steroid-binding proteins in endocrine functions, including hypothalamic-pituitary-adrenal (HPA) axis activity and regulation, as they influence bioavailability, local delivery, and cellular signal transduction of steroid hormones. In the plasma, glucocorticoids (GCs) are mainly bound to the corticosteroidbinding globulin (CBG) and to a lesser extend to albumin. Plasma CBG levels are therefore involved in the adaptive stress response, as they determine the concentration of free, biologically active GCs. In this study, we investigated whether male mice with a genetic predisposition for high-reactivity (HR), intermediate-reactivity (IR), or low-reactivity (LR) stress-induced corticosterone (CORT) secretion present different levels of free CORT and CORT-binding proteins, basally and in response to stressors of different intensity. Our results suggest a fine control interaction between plasma CBG expression and stress-induced CORT release. Although plasma CBG levels, and therefore CBG binding capacity, were higher in HR animals, CORT secretion overloaded the CBG buffering function in response to stressors, resulting in clearly higher free CORT levels in HR compared with IR and LR mice (HR $>I R>L R$ ), resembling the pattern of total CORT increase in all three lines. Both stressors, restraint or forced swimming, did not evoke fast CBG release from the liver into the bloodstream and therefore $C B G$ binding capacity was not altered in our three mouse lines. Thus, we confirm CBG functions in maintaining a dynamic equilibrium between CBG-bound and unbound CORT, but could not verify its role in delaying the rise of plasma free CORT immediately after stress exposure.
\end{abstract}

\author{
Key Words \\ - corticosteroids \\ - HPA axis \\ - hormone transport \\ - mouse \\ - behavior
}




\section{Introduction}

Steroid hormones such as glucocorticoids (GCs), i.e. cortisol and corticosterone (CORT), and sex hormones, e.g. estradiol, progesterone, and testosterone, are generally transported in the blood, bound to carrier proteins such as albumin, sex hormone-, and corticosteroid-binding globulin (CBG, also called transcortin). CBG is a monomeric glycoprotein synthesized and stored mainly by the liver (Rothschild et al. 1972, Weiser et al. 1979, Kuhn et al. 1986, Hammond et al. 1987, Henley \& Lightman 2011). Evidence for an intracellular location of CBG in the CNS and the pituitary has been reported, but its role within the brain remains to be determined (de Kloet et al. 1984, Mopert et al. 2006, Sivukhina et al. 2012). CBG is capable of binding GC with high affinity but with low capacity, in contrast to albumin, which binds GC with high capacity but with low affinity (Slaunwhite \& Sandberg 1959, Sandberg et al. 1964, Burton \& Westphal 1972, Breuner $\&$ Orchinik 2002, Moisan 2010). The association of steroids with CBG is more than three orders of magnitude tighter than with albumin, and the interaction is highly responsive to temperature but not to $\mathrm{pH}$ variations (Cameron et al. 2010). While 80-90\% of circulating GCs are bound to CBG, 10-15\% are bound to albumin, leaving only about $5 \%$ of biologically active unbound free GC available to diffuse through cell membranes and to bind to glucocorticoid receptors and mineralocorticoid receptors (Mendel 1989, Lewis et al. 2005, Henley \& Lightman 2011, Cizza \& Rother 2012). Despite the relatively weak binding affinity of GC to albumin, the physiological concentrations of albumin are such that it must be taken into consideration. The distribution between CBG-bound GC, albumin-bound GC and the free GC fraction in the plasma is challenged when a surge of GC is released from the adrenal cortex in response to stressors. Although only free GCs are able to exert biological effects, little is known about this regulation during stress.

CBG also plays an important role in GC action. It is discussed that $\mathrm{CBG}$ regulates the amount of free $\mathrm{GC}$ by buffering the elevations of GC during a secretory surge. In contrast, during periods of rest or reduced secretion, CBG provides a reservoir of circulating protein-bound GC that is biologically inactive but becomes active after dissociation of the CBG-GC complex, for example at the sites of inflammation where the serine protease elastase specifically cleaves CBG due to its SERPIN structure (Mendel 1989, Breuner \& Orchinik 2002). Interestingly, CBG acts as a protein thermocouple that is exquisitely sensitive to temperature changes, and here too it will act as a GC delivery molecule that will release cortisol in response to fever or external sources of heat (Cameron et al. 2010). Moreover, CBG regulates GC clearance, as the concentration of $\mathrm{CBG}$ in human plasma correlates inversely with metabolic clearance rates for cortisol, and CBG-deficient patients and CBG knockout mice showed increased GC clearance (Siiteri et al. 1982, Bright 1995, Petersen et al. 2006).

Previous reports have suggested that CBG may have an important role in the stress response. In some studies, following certain types of acute or chronic stressors, CBG expression was found to be downregulated, thereby increasing free GC levels (Neufeld et al. 1994, Fleshner et al. 1995, Spencer et al. 1996). Recently, the analysis of a mouse model of targeted CBG deficiency (CBG knockout) has shown that CBG is necessary for mice to mount a normal CORT response and to display adequate adaptive behaviors (Richard et al. 2010, Minni et al. 2012, Moisan 2013). These studies have highlighted the major role of CBG in maintaining a reservoir of GC readily available in case of emergencies, such as stress responses. Furthermore, Qian et al. (2011) have shown that in rats the rise of free CORT in the target compartments such as the brain and subcutaneous tissue is delayed by 20-30 min compared with the total CORT rise in the blood. Moreover, they discovered that CBG is a key player in this delay, as both moderate and strong stressors (i.e. restraint and forced swimming) evoked a fast and significant rise in $\mathrm{CBG}$ protein and binding capacity in the blood through a release of $\mathrm{CBG}$ from the liver. Thus, the increase in circulating CBG levels restrains the rise of free CORT concentrations for $\sim 20 \mathrm{~min}$ in face of mounting total GC levels in the circulation. These results suggest a highly dynamic role for $\mathrm{CBG}$ in regulating free $\mathrm{GC}$ concentrations in response to acute stressors.

Considering the critical role of steroid-binding proteins in mounting adequate neuroendocrine and behavioral responses to stressors, and their eminent role in CORT bioavailability, local delivery, and cellular signal transduction, the overall aim of this study was to investigate whether mice with a genetic predisposition for extremes in HPA axis reactivity, and therefore in the stress-induced secretion of CORT, would differ in the expression and release of free CORT and CORT-binding proteins, i.e. albumin and $\mathrm{CBG}$, in the liver and in plasma, both basally and in response to stressors. To achieve this, we used the recently established stress reactivity (SR) mouse model (Touma et al. 2008). This genetic animal model of affective

Published by Bioscientifica Ltd 
disorders consists of three mouse lines selectively bred for high reactivity (HR), intermediate reactivity (IR) or low reactivity (LR) of the HPA axis in response to stressors.

\section{Materials and methods}

\section{Animals and housing conditions}

From weaning until 4 weeks of age, mice were housed in same-sex groups of four. Housing and experimental rooms were maintained under standard laboratory conditions $(12 \mathrm{~h}$ light: $12 \mathrm{~h}$ darkness cycle, lights on at $0800 \mathrm{~h}$; temperature, $22 \pm 1{ }^{\circ} \mathrm{C}$; relative humidity, $\left.55 \pm 10 \%\right)$. Commercial mouse diet (Altromin no. 1324, Altromin GmbH, Lage, Germany) and tap water were available and the animals were allowed to eat and drink ad libitum. In total, 132 male mice derived from generations XV, XVI, and XX of the HR, IR, and LR breeding lines of the SR mouse model (Touma et al. 2008) were used in this experiments.

In order to avoid potential litter effects, the mice were selected from a minimum of six different litters per line. Animals were between 3 and 5 months of age during the experiments. All experiments were performed during the trough of the circadian rhythm of GC secretion (between 0900 and $1200 \mathrm{~h}$, Touma et al. (2009)) and the order of testing was counterbalanced across the different breeding lines. At least 10 days before the experiments, the animals were housed singly in transparent polycarbonate cages (standard Makrolon cages type II, Bayer MaterialScience $\mathrm{GmbH}$, Darmstadt, Germany; $23 \times 16 \times 14 \mathrm{~cm}$ ). The presented work complies with current regulations covering animal experimentation in Germany and the EU (European Communities Council Directive 86/609/EEC). All experiments were announced to the appropriate local authority and were approved by the 'Animal Welfare Officer' of the Max Planck Institute of Psychiatry (Az. 55.2-1-54-2531-64-07).

\section{Experimental designs}

To investigate whether genetic alterations of HPA axis reactivity, i.e. dysregulated stress-induced CORT secretion, would interfere with the expression and release of free CORT and CORT-binding proteins, we performed three sets of experiments (see below). The experimental design is also depicted in Supplementary Figure 1, see section on supplementary data given at the end of this article.

Experiment 1: basal and restraint-stress-induced total CORT, CBG, and albumin To assess total CORT, CBG, and albumin concentrations in the plasma and liver under basal conditions and after stress exposure, a total of 54 male mice were subjected to a psychological stressor in the SR test (SRT). The SRT consists of a 15-min restraint period and two tail blood samples immediately before $(t=0)$ and after restraint $(t=15)$ (Touma et al. 2008). After the SRT, animals were transferred back to their home cages for $15 \mathrm{~min}$. Thirty minutes after the beginning of the restraint period $(t=30)$, the mice $(n=10$ per line) were decapitated after isofluorane anesthesia and trunk blood was collected. Livers were carefully dissected without the gall bladder, immediately frozen on dry ice and stored at $-80^{\circ} \mathrm{C}$ until further analysis. Animals of the basal group ( $n=8$ per line) were killed under unstressed conditions. Trunk blood and livers were harvested following the same protocol. All blood samples were treated with the protease inhibitor, Trasylol (Bayer Vital $\mathrm{GmbH}$, Leverkusen, Germany), centrifuged for $10 \min \left(4000 \boldsymbol{g}\right.$ at $\left.4^{\circ} \mathrm{C}\right)$, and the plasma was stored at $-80^{\circ} \mathrm{C}$ until further analysis, when plasma total CORT concentrations were determined (see section 'Plasma total CORT'). Plasma and liver samples were used for CBG and albumin immunoblottings (see section 'Immunoblotting').

\section{Experiment 2: basal and restraint-stress-induced} free CORT and CBG Bmax To evaluate plasma free CORT and CBG maximal binding capacity (CBG Bmax) in unstressed and stressed animals, 24 male mice $(n=8$ per line) were subjected to the same $15 \mathrm{~min}$ restraint stressor as in Experiment 1. Immediately after restraint $(t=15)$, animals were decapitated and trunk blood was collected. Animals of the basal group ( $n=8$ per line) were killed under unstressed conditions; trunk blood was collected following the same protocol as described above. Plasma free CORT concentrations and CBG Bmax were determined (see sections 'Plasma free CORT' and 'CBG binding capacity').

Experiment 3: total CORT, free CORT, CBG, and albumin in response to forced swimming In order to assess total and free CORT, CBG, and albumin levels before and after exposure to a more severe (physical+ psychological) stressor, 30 male mice ( $n=10$ per line) were subjected to a 10-min open field (OF) test, directly followed by a 6-min forced swim test (FST). The animals were killed by decapitation immediately after the FST and trunk blood was collected as described earlier. Three days before the start of the experiment, a basal blood sample of each animal was obtained by tail nick. Plasma samples were used for CBG and albumin immunoblotting and total

Published by Bioscientifica Ltd. 
and free CORT measurements (see sections 'Plasma total CORT' and 'Immunoblotting').

The OF test and FST are described in detail elsewhere (Touma et al. 2008). Briefly, the OF apparatus consisted of a black, open topped PVC cylinder $(60 \mathrm{~cm}$ diameter and $40 \mathrm{~cm}$ height), and was dimly illuminated (15 lux). The animals' locomotor activities and exploratory drives were assessed utilizing the ANY-maze video tracking software (Stoelting Co., Wood Dale, IL, USA). In the FST, the animals were placed in a glass beaker filled with $23^{\circ} \mathrm{C}$ water, where they are unable to reach the bottom with their hind paws or tail. The duration of struggling, swimming, and floating behavior was scored by a trained observer blind to mouse line using the EVENTLOG Software (Event Recorder, EMCO Maier GmbH, Hallein-Taxach, Austria).

\section{Neuroendocrine measures}

Plasma total CORT Plasma total CORT concentrations were measured in duplicate using a commercially available CORT RIA Kit (DRG Instruments GmbH, Marburg, Germany) following the manufacturer's protocol with slight modifications as described previously (Touma et al. 2008). The intra and inter-assay coefficients of variation $(\mathrm{CV})$ were both below $10 \%$.

Plasma free CORT Plasma free CORT was estimated by isotopic dilution and plasma ultrafiltration as described previously (Minni et al. 2012). Free CORT percentage was calculated as the ratio of c.p.m. filtrate (free CORT)/c.p.m. retentate (bound CORT). Free CORT concentration was obtained as percentage of free CORT multiplied by total CORT concentration measured by prior RIA.

CBG binding capacity $C B G$ maximal binding capacity (Bmax) was assessed by a saturation binding assay as described previously (Hammond \& Lahteenmaki 1983, Richard et al. 2010). The intra-assay CV was below $10 \%$. All samples from an experiment were measured in the same assay to avoid inter-assay variation.

\section{Immunoblotting}

Whole livers were homogenized for 2 min using a tissue homogenizer (VDI 12, VWR International GmbH, Darmstadt, Germany) in $5 \mathrm{ml}$ ice-cold RIPA buffer (50 mM Tris$\mathrm{HCl}, \mathrm{pH} 8.0,150 \mathrm{mM} \mathrm{NaCl}, 0.1 \%$ SDS, $1 \% \mathrm{NP}-40$, and $0.5 \%$ sodium deoxycholate) with protease inhibitors (Complete Mini Protease Inhibitor Cocktail Tablets, Roche Applied Sciences). Homogenates were then sonified with 20 strokes (Branson Sonifier 250, G. Heinemann, Schwäbisch Gmünd, Germany). The samples were subsequently incubated with continuous rolling for $30 \mathrm{~min}$ at $4{ }^{\circ} \mathrm{C}$ to accomplish cell lysis. Homogenates were centrifuged for $30 \mathrm{~min}\left(11350 \mathrm{~g}\right.$ at $\left.4^{\circ} \mathrm{C}\right)$ and the transparent supernatants were saved. Protein concentration was quantified in triplicates using a BCA protein assay (Thermo Scientific, Rockford, IL, USA) read at $550 \mathrm{~nm}$ (Dynatech MR 7000, (Dynatech, Denkendorf, Germany)).

Ten nanograms to ten micrograms of protein (depending on the target protein) were separated by $10 \%$ SDS-PAGE and transferred onto nitrocellulose membranes (Whatman GmbH, Dassel, Germany). The membranes were blocked for $1-2 \mathrm{~h}$ in 5\% (w/v) fat-free milk (Carl Roth $\mathrm{GmbH}$, Karlsruhe, Germany) in Tris-buffered saline 0.1\% Tween 20 (TBST). Primary antibodies were diluted in $2.5 \%$ milk-TBST solution and incubated overnight at $4{ }^{\circ} \mathrm{C}$ : rabbit anti-human CBG 1:2000 (Affiland (Liege, Belgium), as previously applied in rodents studies (Qian et al. 2011)), rabbit anti-mouse albumin 1:50 000 (Cedarlane, Burlington, ON, Canada), mouse anti-glyceraldehyde-3-phosphate-dehydrogenase 1:2000, and rabbit anti-TF 1:1000 (Santa Cruz Biotechnology Inc., Santa Cruz, CA, USA). Following incubation with the respective HRP-conjugated secondary antibody anti-rabbit or anti-mouse (SigmaAldrich and Jackson ImmunoResearch Europe Ltd., Newmarket, Suffolk, UK) for $1 \mathrm{~h}$ at room temperature, membranes were incubated with ECL solution, protein bands were detected by the ChemiDoc MP imaging system (Bio-Rad Laboratories $\mathrm{GmbH}$ ) and analyzed by Image Lab Software (version 4.0.1 build 4, Bio-Rad).

\section{Statistical analysis}

As normality and variance homogeneity could not always be assumed, all data were exclusively analyzed using nonparametric statistics (Siegel \& Castellan 1988). All tests were applied two-tailed and were calculated using the software package SPSS (version 18.0). Two dependent samples were compared using the Wilcoxon test ( $W$-test). Differences between more than two dependent samples were calculated with the Friedman test ( $F$-test). Two independent samples were compared using the MannWhitney $U$ test (MWU-test), while differences between more than two independent samples were calculated with the Kruskal-Wallis $H$-test (KWH-test). In the case of significant variation proven by the $F$ - or KWH-tests, post-hoc pairwise comparisons between the groups were done using multiple $W$ - or MWU-tests respectively. Here, significance levels were corrected according to the

Published by Bioscientifica Ltd. 
sequential Bonferroni's technique (Rice 1989). Differences were considered significant if their probability of occurring by chance was $<5 \%(P \leq 0.05)$.

\section{Results}

\section{Free CORT mirrors total CORT in response to restraint stress}

Plasma samples were obtained from animals under basal conditions or after restraint, according to the methods for Experiments 1 and 2 (see sections 'Experiment 1: basal and

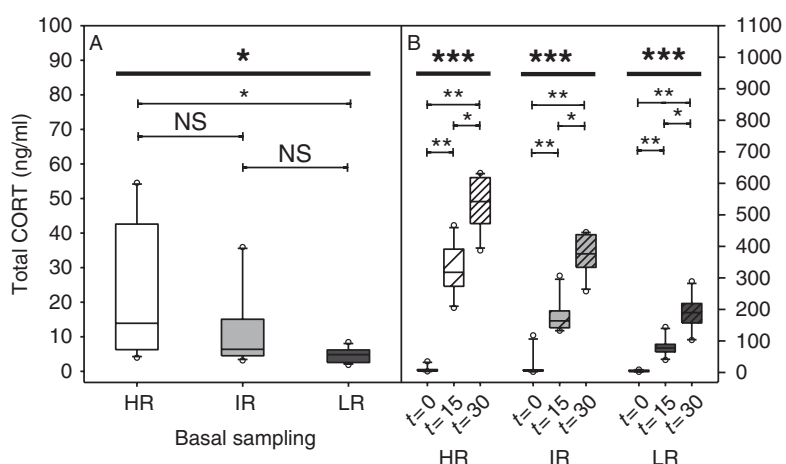

restraint-stress-induced total CORT, CBG, and albumin' and 'Experiment 2: basal and restraint-stress-induced free CORT and CBG Bmax'; Supplementary Figure 1). As expected, plasma total CORT concentrations were low in all three lines of the basal group, although a between-line comparison showed that HR animals had significantly higher total CORT concentrations compared with mice of the LR line (Fig. 1A). Similarly, in the blood sample collected immediately before restraint $(t=0)$, plasma total CORT concentrations were low (Supplementary Figure 2A, see section on supplementary data given at the end of this article), but restraint ( $t=15)$ markedly increased plasma
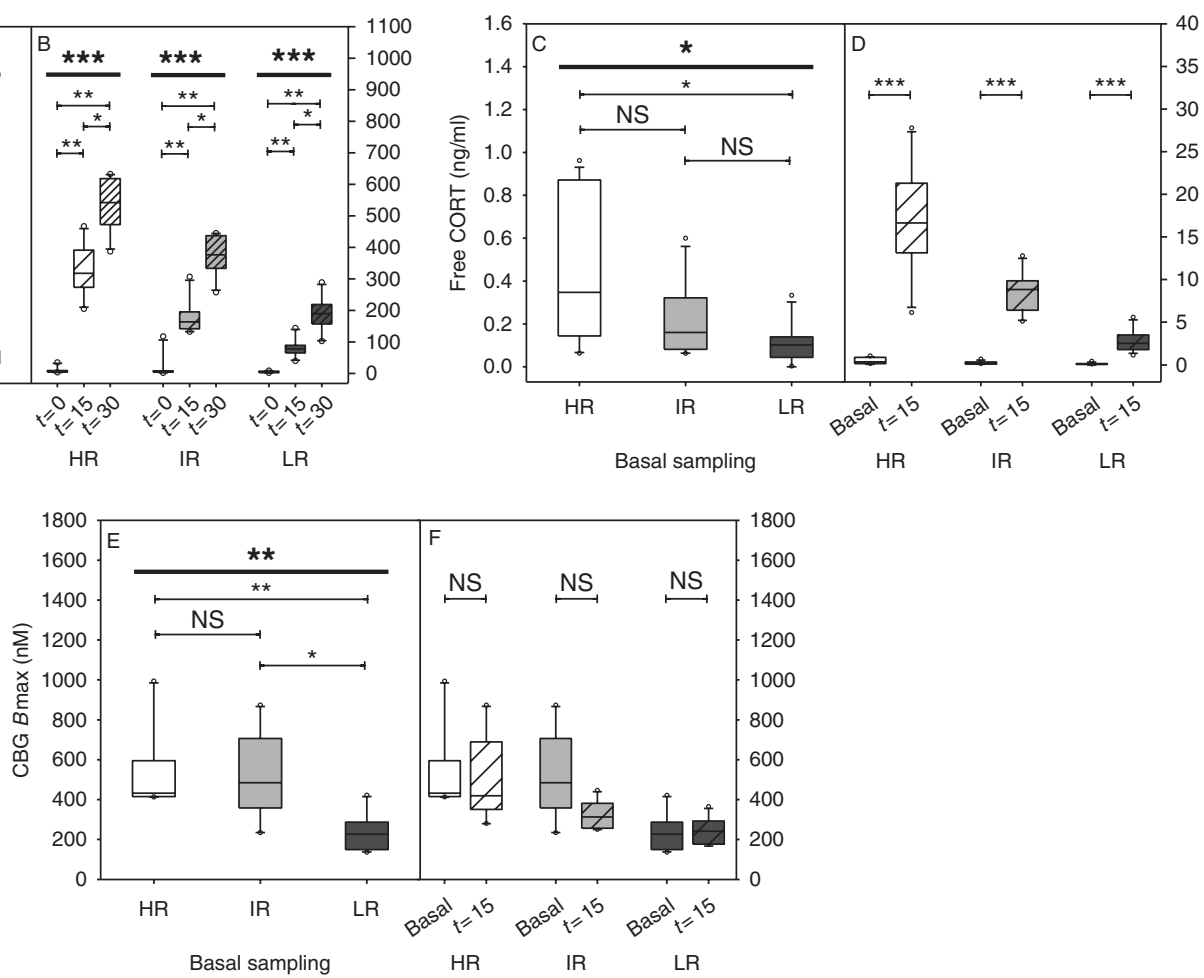

\section{Figure 1}

Effect of 15 min restraint on total and free corticosterone (CORT) concentrations and corticosteroid-binding globulin (CBG) binding capacity (Bmax) in the plasma of high (HR), intermediate (IR), and low (LR) reactivity mice. Data are given as box plots showing medians (lines in the boxes), 25 and $75 \%$ percentiles (boxes), 10 and $90 \%$ percentiles (whiskers) as well as outliers (circles). (A) Plasma total CORT levels under basal conditions (KWH-test basal $: n=8 / 8 / 8, H=6.8, \mathrm{df}=2, P=0.032$; post-hoc MWU-tests basal $^{\text {: }}$ $H R$ vs $I R, U=19, P=0.172$; HR vs $L R, U=9, P=0.048$; and $I R$ vs $L R, U=17$, $P=0.230$ ). (B) $15-\mathrm{min}$ restraint stress $(t=15)$ caused a significant increase in total CORT levels in the plasma, total CORT kept increasing to significantly higher levels up to $30 \mathrm{~min}(t=30)$ after the onset of restraint ( $F$-test te $_{t=0}$ vs $t=15$ vs $30: \mathrm{HR} / \mathrm{IR} / \mathrm{LR}, n=10 / 10 / 10$, all $\chi^{2}=20$, all $\mathrm{df}=2$, all $P \leq 0.001$; post-hoc $W$-tests: $t=0$ vs 15 , all $Z=-2.8$, all $P=0.01 ; t=0$ vs 30 , all $Z=-2.8$, all $P=0.005$; and $t=15$ vs 30 , all $Z=-2.8$, all $P=0.015$ ). (C) Plasma free CORT concentrations under basal conditions (KWH-test basal: $n=8 / 8 / 8$, $H=7.0, \mathrm{df}=2, P=0.030$; post-hoc MWU-tests basal: $\mathrm{HR}$ vs $\mathrm{IR}, U=17, P=0.230$; HR vs $L R, U=9, P=0.048$; and IR vs $L R, U=18, P=0.141$ ). (D) Free CORT levels followed a similar pattern to total CORT in response to the 15-min restraint stressor (MWU-tests: $\mathrm{HR}_{\text {basal }}$ vs $H R_{t=15}, \mathrm{IR}_{\text {basal }}$ vs $\mathrm{IR}_{t=15}, \mathrm{LR}_{\text {basal }}$ vs $\mathrm{LR}_{t=15}$, all $U=0$, all $P=0.001$ ). (E) CBG binding capacity (CBG $B$ max) was significantly lower in LR mice under basal conditions (KWH-test basal: $n=8 / 8 / 8, H=12.2, \mathrm{df}=2, P=0.002$; post-hoc MWU-tests basal $_{\text {: }}$ HR vs IR, $U=31, P=0.916$; HR vs $L R, U=1, P=0.003$; and IR vs $L R, U=6, P=0.012$ ). (F) CBG Bmax was not significantly changed by the 15 -min restraint stressor (MWU-tests: $\mathrm{HR}_{\text {basal }}$ vs $\mathrm{HR}_{t=15}, U=24, P=0.401$; $\mathrm{IR}_{\text {basal }}$ vs $\mathrm{IR}_{t=15}, U=13$, $P=0.083$; and $\mathrm{LR}_{\text {basal }} \mathrm{Vs} \mathrm{LR}_{t=15}, U=26, P=0.529$ ). Basal, unstressed animals; $t=0$, sampling immediately before restraint; $t=15$, sampling immediately after the restraint; $t=30$, sampling 30 min after beginning of restraint stressor. Significant differences are indicated at the top of each panel by bold lines and bold symbols (KWH- or F-tests), pairwise group comparisons (post-hoc MWU- or $W$-tests) are indicated below with thinner lines spanning between the two compared groups $(P>0.05 \mathrm{NS}, * P \leq 0.05$, $* * P \leq 0.01$, and $* * * P \leq 0.001)$. http://joe.endocrinology-journals.org DOI: $10.1530 / J O E-13-0255$
(C) 2013 Society for Endocrinology Printed in Great Britain 
total CORT concentrations in all three mouse lines, and CORT levels were even further elevated $30 \mathrm{~min}(t=30)$ after the onset of the restraint stressor (Fig. 1B). Furthermore, there was a significant difference in the magnitude of the CORT increase between the three lines, both at $t=15$ and 30 , with HR mice displaying a greater rise in
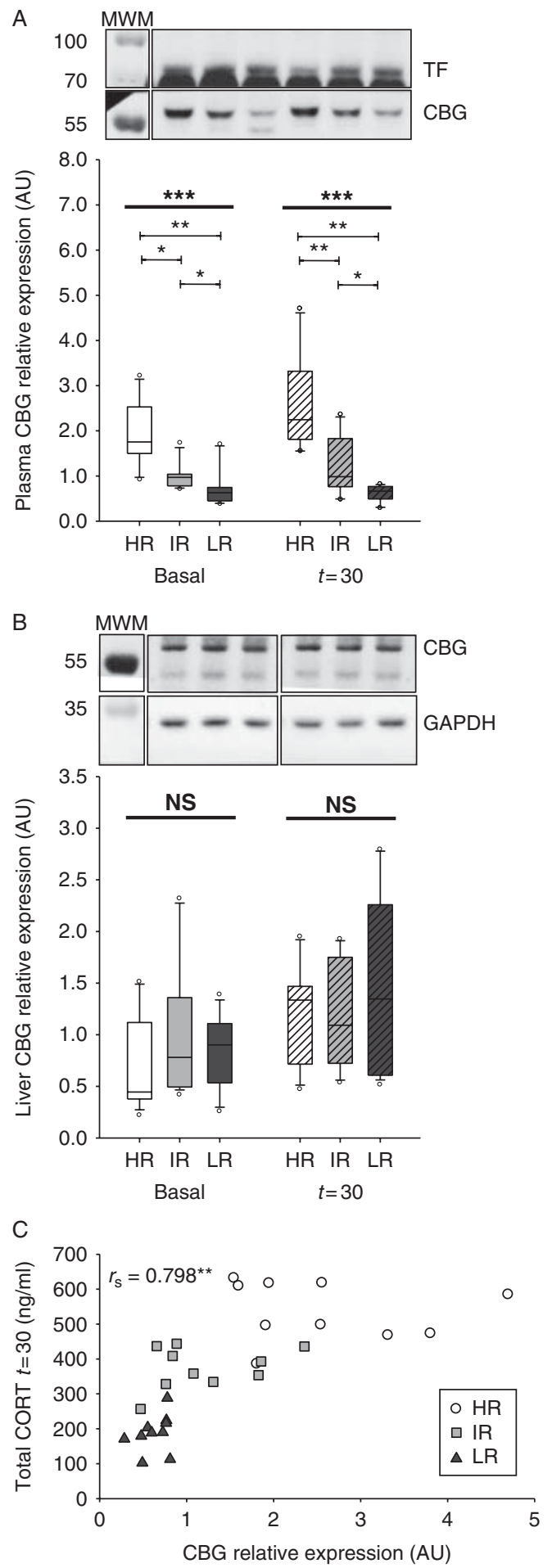

http://joe.endocrinology-journals.org DOI: 10.1530/JOE-13-0255 plasma total CORT concentrations than that of IR mice, while LR animals showed the lowest increase (HR $>$ IR $>$ LR, Supplementary Figure 2B).

Similar to the total CORT measurements, plasma free CORT levels were low in all three lines of the basal group and significant differences could be detected between HR and LR animals (HR $>$ LR, Fig. 1C). After the 15-min restraint $(t=15)$, plasma free CORT concentrations were significantly increased in all three mouse lines (Fig. 1D). As observed for plasma total CORT, there was a significant difference in the magnitude of the free CORT increase between the three lines (HR $>I R>L R$, Supplementary Figure 2C). CBG Bmax also differed between the three lines. It was significantly lower in LR mice under basal conditions (Fig. 1E), as well as after the restraint stressor $(t=15)$ (Supplementary Figure 2D). Stress exposure did not affect CBG Bmax in any of the lines (Fig. 1F).

\section{Differential expression of CBG but not albumin}

Plasma and liver samples from restrained animals were analyzed as described for Experiment 1 (see section 'Experiment 1: basal and restraint-stress-induced total CORT, CBG, and albumin'; Supplementary Figure 1).

\section{Figure 2}

Effects of restraint stress on corticosteroid-binding globulin (CBG) protein expression in the plasma and liver of high (HR), intermediate (IR), and low $(\mathrm{LR})$ reactivity mice. Representative western blot images of $C B G$, transferrin (TF), and glyceraldehyde-3-phosphate-dehydrogenase (GAPDH) in the plasma (A) and liver (B) of mice killed under unstressed conditions (basal) or $30 \mathrm{~min}$ after the onset of a 15-min restraint stressor $(t=30)$ are presented above each panel. Data are given as box plots (for description see legend of Fig. 1). (A) CBG plasma concentrations were significantly different between the three mouse lines under both conditions (KWH-tests: HR/IR/LR $R_{\text {basal, }}$ $n=8 / 7 / 8, H R / R_{R} / L_{t=30}, n=10 / 10 / 10, H=13.6-18.9, \mathrm{df}=2$, all $P \leq 0.001$; post-hoc MWU-tests: basal, HR vs IR, $U=5, P=0.016$; HR vs $L R, U=3$, $P=0.006$; IR vs $\mathrm{LR}, U=9, P=0.028 ; t=30$, HR vs IR, $U=11, P=0.006$; HR vs $\mathrm{LR}$, $U=0, P=0.003$; and IR vs $L R, U=18, P=0.016$ ). The strong band observed bellow TF is probably albumin, which due to its high abundance was constantly present in the TF blots but did not interfere with TF quantification. (B) CBG protein expression in the liver was not different between the lines under basal conditions and remained unchanged after restraint (KWH-tests: all HR/IR/LR, $n=8 / 8 / 8$, all $\mathrm{df}=2$, basal: $H=2.7$, $P=0.249 ; t=30: H=0.1, P=0.949)$. The expression of $C B G$ relative to the sample loading controls (TF or GAPDH) was normalized to the mean of the IR group. Significant differences between the three lines are indicated at the top of each panel by bold lines and bold symbols (KWH-test), pairwise group comparisons (post-hoc MWU-tests) are indicated below with thinner lines spanning between the two compared groups $\left(P>0.05 \mathrm{NS},{ }^{*} P \leq 0.05\right.$, $* * P \leq 0.01$, and $* * * P \leq 0.001)$. Molecular weights $(\mathrm{kDa}): \mathrm{CBG}=55, \mathrm{TF}=79$, $\mathrm{GAPDH}=37, \mathrm{MWM}$, molecular weight marker. (C) Correlation between plasma CBG relative protein expression and plasma total CORT levels $30 \mathrm{~min}$ after the onset of a 15 -min restraint stressor $(t=30)$. Spearman's rank correlation coefficient $\left(r_{\mathrm{s}}\right), n=10 / 10 / 10, * * P \leq 0.01$.

Published by Bioscientifica Ltd. 
The three mouse lines differed significantly regarding their plasma CBG protein expression in both the basal and the stressed groups (Fig. 2A). HR mice showed clearly increased plasma CBG levels compared with IR animals,
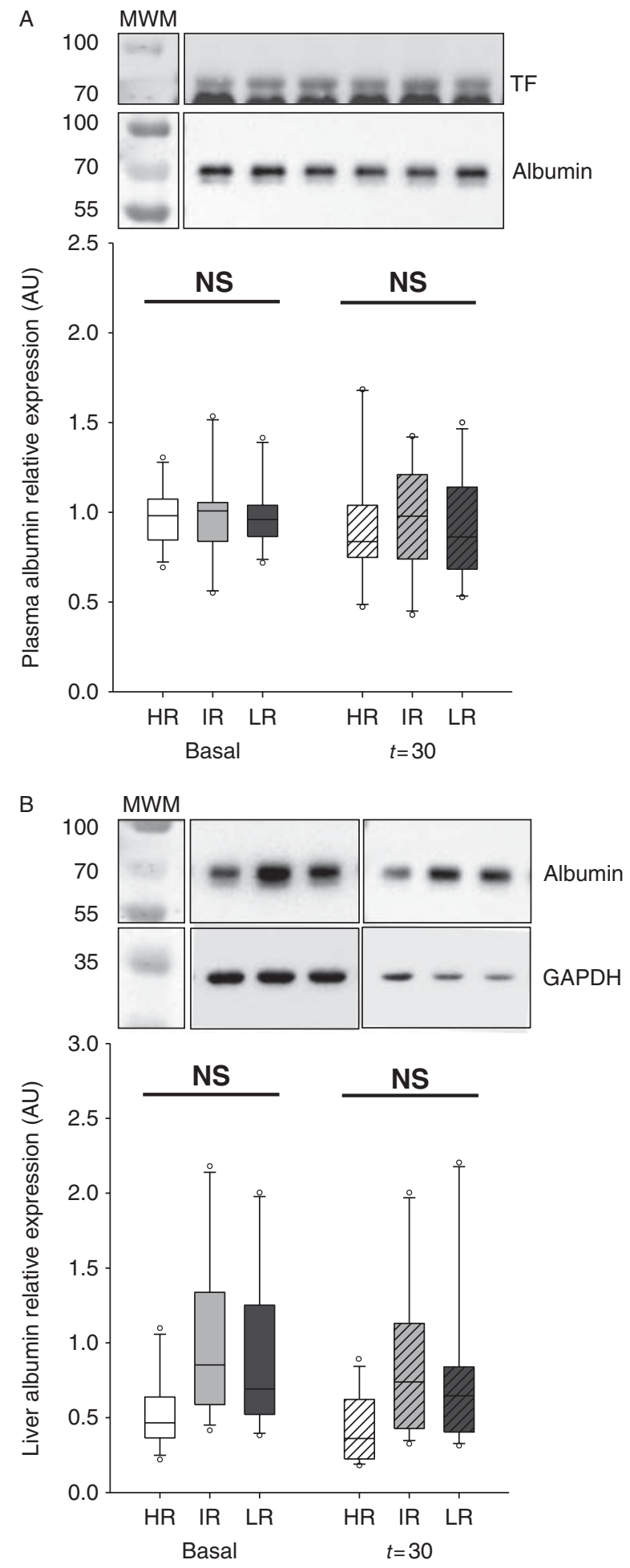

http://joe.endocrinology-journals.org DOI: 10.1530/JOE-13-0255 while animals of the LR line presented the lowest amount of plasma CBG (Fig. 2A). Moreover, a positive correlation was found between plasma CBG protein expression and plasma total CORT levels $30 \mathrm{~min}$ after the onset of the restraint stressor (Fig. 2C).

The within-line comparison, however, did not reveal significant differences between basal and restrained animals of either line (Supplementary Figure 3A, see section on supplementary data given at the end of this article). The analysis of CBG expression in the liver revealed no significant differences between HR, IR, and LR mice (Fig. 2B). Furthermore, there was no significant effect of restraint stress on liver CBG expression in IR and LR mice (Supplementary Figure 3B). Similarly, the expression of albumin did not differ significantly neither in the plasma (Fig. 3A) nor in the liver (Fig. 3B) of HR, IR, and LR animals under basal conditions as well as after restraint stress exposure (Supplementary Figure 4A and B, see section on supplementary data given at the end of this article).

\section{Psychological and physical stressors do not modulate CBG plasma levels}

Plasma samples collected after 15 -min restraint (SRT, Experiment 1) or after exposure to the FST (Experiment 3) were analyzed (see sections 'Experiment 1: basal and restraint stress-induced total CORT, CBG, and albumin', and 'Experiment 3: total CORT, free CORT, CBG, and albumin in response to forced swimming'; Supplementary

\section{Figure 3}

Effects of restraint stress on albumin protein expression in the plasma and liver of high (HR), intermediate (IR), and low (LR) reactivity mice. Representative western blot images of albumin, transferrin (TF), and glyceraldehyde-3-phosphate-dehydrogenase (GAPDH) in the plasma (A) and liver (B) of mice killed under unstressed conditions (basal) or $30 \mathrm{~min}$ after the onset of a 15-min restraint stressor $(t=30)$ are presented above each panel. Data are given as box plots (for description see legend of Fig. 1). (A) Albumin concentrations in the plasma (KWH-tests: HR/IR/LR $\mathrm{Basal}_{\text {, }}$ $n=8 / 7 / 7, H=0.3, \mathrm{df}=2, P=0.826$; and $\mathrm{HR} / \mathrm{IR} / \mathrm{LR}_{t=30}, n=10 / 10 / 10, H=0.1$, $\mathrm{df}=2, P=0.946)$ and (B) in the liver (KWH-tests: all $n=8 / 8 / 8, H R / \mathrm{R}^{\prime} / \mathrm{LR}_{\text {basal, }}$ $H=5.4, \mathrm{df}=2, P=0.064 ;$ and $\mathrm{HR} / \mathrm{R} / \mathrm{LR}_{t=30}, H=4.8, \mathrm{df}=2, P=0.087$ ) were not significantly different between the three mouse lines under basal conditions and also remained not significantly different between the lines after exposure to the restraint stressor. The strong band observed below TF is probably albumin, that due to its high abundance was constantly present in the TF blots but did not interfere with TF quantification. The expression of albumin relative to the sample loading controls (TF or GAPDH) was normalized to the mean of the IR group. Significant differences between the three lines are indicated at the top of each panel by bold lines and bold symbols (KWH-test) $(P>0.05 \mathrm{NS})$. Molecular weights (KDa): albumin, 69; TF, 79; GAPDH, 37; MWM, molecular weight marker. 


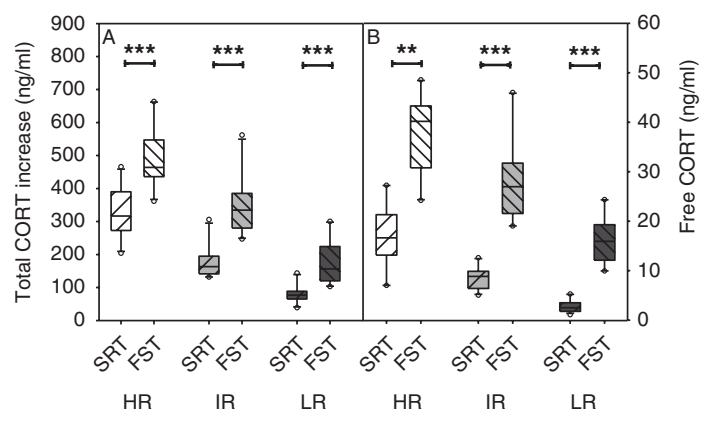

Figure 4

Effects of different stressors on total corticosterone (CORT) increase free CORT levels and CBG and albumin protein expression in the plasma of high $(H R)$, intermediate (IR), and low (LR) reactivity mice. Representative western blot images of CBG, albumin, and transferrin (TF) in the plasma from mice sampled under unstressed conditions (basal), after 15-min restraint stress (SRT), and after a 10-min open field test plus 6-min forced swim test (FST). Data are given as box plots (for description see legend of Fig. 1). (A) Plasma total CORT increases after the FST was significantly higher than after the SRT (MWU-tests: for each line $n=10 / 10, H_{S R T}$ VS HR $R_{F S T} U=8$, $P=0.001 ; \operatorname{IR}_{\mathrm{SRT}}$ vs $\mathbb{I R}_{\mathrm{FST}}, U=0, P \leq 0.001$; and $L_{\mathrm{SRT}}$ Vs $L_{\mathrm{FST}}, U=5, P=0.001$ ). (B) Plasma free CORT after the FST was significantly higher than after the SRT (MWU-tests: HR $\mathrm{SRT}_{\mathrm{ST}}$ Vs HR $\mathrm{FR}_{\mathrm{FST}}, n=8 / 7, U=1, P=0.002 ; \mathrm{IR}_{\mathrm{SRT}}$ vs IR $\mathrm{R}_{\mathrm{FST}}, n=7 / 8$, $U=0, P=0.001$; and $\mathrm{LR}_{\mathrm{SRT}}$ Vs $\mathrm{LR}_{\mathrm{FST}}, n=8 / 8, U=0, P=0.001$ ). (C) CBG expression was significantly higher in the plasma of HR mice compared with IR and/or LR animals for all three conditions (KWH-test: HR/IR/LR $\mathrm{R}_{\text {basal }}$ : $n=5 / 5 / 5, \mathrm{df}=2, H=7.4, P=0.024$; and HR/R/LR $\mathrm{SRT}: n=5 / 5 / 5, \mathrm{df}=2, H=6.6$,

Figure 1). As expected, plasma total and free CORT values after the FST were significantly higher than those after the SRT (Fig. 4A and B). However, plasma CBG levels after the FST remained similar to those observed under basal conditions and after the SRT (Fig. 4C and Supplementary Figure 5C). A between-line comparison revealed significant differences in the swim-stress-induced plasma total and free CORT concentrations, with HR mice showing clearly higher plasma total and free CORT levels compared with the IR line, while LR animals presented the lowest CORT increase (Supplementary Figure 5A and B). Regarding plasma albumin expression, no differences were found between the three mouse lines under basal conditions, as well as after stress exposure in the SRT or FST (Fig. 4D). The within-line comparison revealed a tendency toward increased albumin levels after the FST only in HR animals (Supplementary Figure 5D).

Regarding the behavioral response of the animals in the OF test and the FST, significant differences were observed between HR, IR, and LR mice. The results of the OF test assessing the locomotor activity and exploratory drive are summarized in Supplementary Table 1, see section on supplementary data given at the end of this

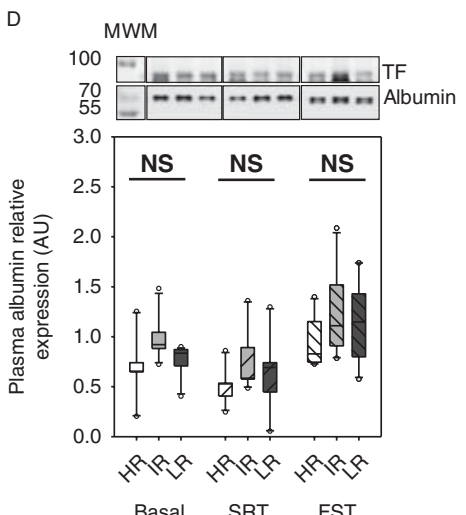

$P=0.037 ; \mathrm{HR} / \mathrm{IR} / \mathrm{LR}_{\mathrm{FST}}: n=10 / 10 / 10, \mathrm{df}=2, H=7.1, P=0.028 ;$ post-hoc MWU-tests: basal, HR vs IR, $U=3, P=0.094 ; \mathrm{HR}$ vs $\mathrm{LR}, U=0, P=0.027$; IR vs LR, $U=11, P=0.754$; SRT, HR vs IR, $U=3, P=0.094$; HR vs LR, $U=1, P=0.016$; IR vs LR, $U=11, P=0.754$; FST, HR vs IR, $U=14, P=0.021$; HR vs $L R, U=29$, $P=0.224$; and IR vs $L R, U=39, P=0.406$ ). (D) Albumin plasma concentrations were not significantly different between the lines for all three conditions (KWH-test: HR/IR/LR basal $n=5 / 5 / 5, \mathrm{df}=2, H=5.0, P=0.080$; $\mathrm{HR} / \mathrm{IR} / \mathrm{LR}_{\mathrm{SRT}}: n=5 / 5 / 5, \mathrm{df}=2, H=2.0, P=0.368$; and HR/IR/LR $\mathrm{FST}: n=10 / 10 / 10$, $\mathrm{df}=2, H=2.7, P=0.247)$. The $C B G$ and albumin relative expressions to the sample loading control (transferrin (TF)) were normalized to the mean of the IR group. Significant differences between the three lines are indicated at the top of each panel by bold lines and bold symbols (KWH-test), pairwise comparisons (MWU-tests) are indicated with thinner lines spanning between the two compared groups ( $P>0.05 \mathrm{NS},{ }^{*} P \leq 0.05$, $* * P \leq 0.01$, and $* * * P \leq 0.001)$. Molecular weights (kDa): CBG, 55; albumin, 69; TF, 79; MWM, molecular weight marker.

article. The FST also revealed significant differences in stress-coping behavior between the three lines, with HR animals showing clearly increased struggling and decreased floating compared with IR animals, while LR mice showed more passive coping behavior with less struggling and more floating (Fig. 5A, B and C).

\section{Discussion}

The emerging role of steroid-binding proteins in endocrine functions has received increasing attention (Nakhla et al. 1999, Breuner \& Orchinik 2002, Henley \& Lightman 2011, Perogamvros et al. 2012). Plasma CBG levels are important for the adaptive stress responses as they determine the GC pool size and consequently the concentration of free GC (Minni et al. 2012, Moisan 2013). In this study, three mouse lines selectively bred for HR, IR, or LR stress-induced secretion of CORT were used to elucidate whether the differences in HPA axis reactivity are influenced by differential free CORT and CBG availability and release. We performed experiments involving stressors with different intensity and analyzed plasma and liver samples for the abundance of

Published by Bioscientifica Ltd 


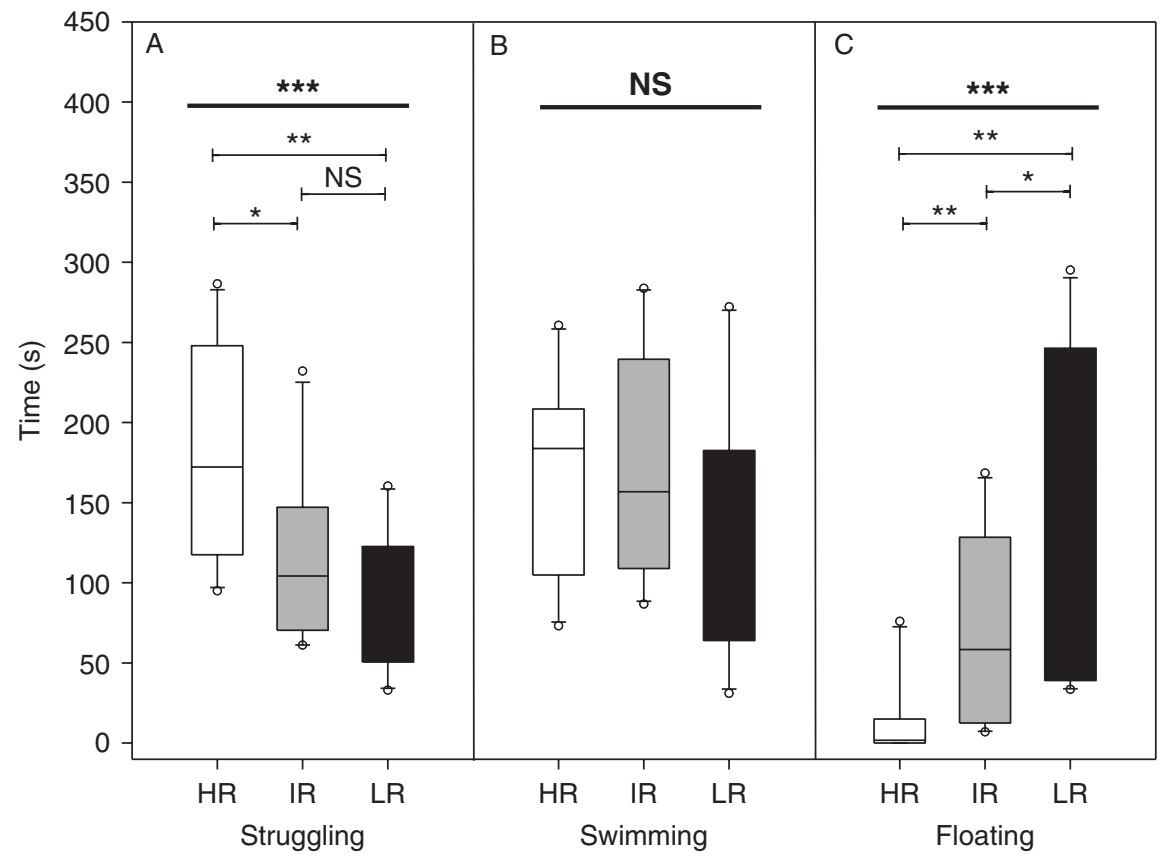

\section{Figure 5}

Stress-coping behavior of high (HR), intermediate (IR), and low (LR) reactivity mice in the forced swim test (FST). Data are given as box plots (for description see legend of Fig. 1). (A) HR mice showed significantly increased struggling time compared with the other two lines. (B) Total swimming time was not significantly different between the three mouse lines. (C) HR mice showed clearly decreased floating behavior, while LR mice show increased floating compared with IR animals. Significant differences between the three lines (KWH-test: all $n=10 / 10 / 10$, all $\mathrm{df}=2$,

CORT, CBG, and albumin. Our results revealed that 15-min restraint as well as 6-min forced swimming did not trigger a fast $\mathrm{CBG}$ release from the liver into the circulation, although we could observe significant line differences regarding plasma total and free CORT as well as plasma CBG expression and CBG Bmax ( $\mathrm{HR}>\mathrm{IR}>\mathrm{LR}$ ).

The currently accepted model of steroid action proposes that only steroids that are not bound to plasma proteins can gain access to target tissues and intracellular receptors (Siiteri et al. 1982, Mendel 1989). Hence, we evaluated plasma free CORT concentrations before and after exposure to restraint stress (Fig. 1C and D). As expected, the stress-induced surge of CORT exceeds CBG's binding capacity, resulting in significantly increased, but different, free plasma CORT concentrations (HR $>$ IR $>$ LR), thus paralleling the line-specific differences in plasma total CORT (see Fig. 1B and D). Interestingly, we were able to demonstrate significantly increased plasma free CORT concentrations as early as $15 \mathrm{~min}$ after stress initiation (Fig. 1D). Heinzmann et al. (2010), using in vivo microdialysis, reported that the rise in free CORT levels in the
HR/IR/LR struggling: $H=13.0, P=0.001 ; H R / / R / L_{\text {swimming }}: H=3.8, P=0.147 ;$ and $\left.\mathrm{HR} / \mathrm{IR} / \mathrm{LR}_{\text {floating }}: H=16.5, P \leq 0.001\right)$ are indicated at the top of the panel by bold lines and bold symbols, pairwise group comparisons (post-hoc MWU-tests: struggling: $H R$ vs $I R, U=17, P=0.013$; $H R$ vs $L R, U=7, P=0.003$; IR vs $L R, U=28, P=0.096$; floating: HR vs $I R, U=10, P=0.004$; HR vs $L R, U=6$, $P=0.003$; and IR vs $\mathrm{LR}, U=19, P=0.019)$ are indicated below with thinner lines spanning between the two compared groups $\left(P>0.05 \mathrm{NS}\right.$, ${ }^{*} \leq 0.05$, $* * P \leq 0.01$, and $* * * P \leq 0.001)$.

hippocampus is delayed for about $30 \mathrm{~min}$ and peaks at around $60 \mathrm{~min}$ after the onset of the restraint in HR, IR, and LR mice. This is in line with other studies showing a delayed increase in free CORT levels in the brain and other GC target tissues in mice (Thoeringer et al. 2007, Tronche et al. 2010) and rats (Droste et al. 2008, 2009, Qian et al. 2011). Recently, Qian et al. (2011) have shown that, in rats, this 20-30 min delay in the increase in free CORT in the plasma (jugular vein), as well as in other body compartments (subcutaneous tissue and hippocampus), is associated with a fast rise of CBG in the plasma in response to stressors, i.e. forced swimming and exposure to novel environment. However, applying a similar experimental design in our SR mice, we observed elevated plasma free CORT concentrations after 15-min of restraint stress without changes in plasma CBG concentrations or CBG binding capacity (see Figs 1D and $\mathrm{F}, 2 \mathrm{~A}$ ). Therefore, it remains to be clarified whether the regulatory interactions of $\mathrm{CBG}$ and CORT are different in mice and rats or whether the timeframe of these physiological processes follows different patterns in these two rodent species. 
It is conceivable that increased CORT clearance might contribute to the low plasma free CORT values observed in LR mice (Fig. 1D), as indicated by results from Cbg knockout (Cbg-/-) animals (Petersen et al. 2006) and humans (Siiteri et al. 1982). Plasma half-life of injected $\left[{ }^{3} \mathrm{H}\right]$ corticosterone in $\mathrm{Cbg}-/-$ animals is significantly shortened, while after $5 \mathrm{~min}$ of injection there was 10\% of the radioactive steroid left in the plasma of WT mice, there was only $2.5 \%$ in $\mathrm{Cbg}-/$ - animals, suggesting a fourfold higher clearance due to the CBG knockout (Petersen et al. 2006). In human plasma, CBG concentrations inversely correlate with metabolic clearance rates of cortisol (Siiteri et al. 1982). Although there is clear evidence indicating that the adrenal size and sensitivity of the CORT-secretion response to ACTH are significantly different between HR, IR, and LR mice (diminished in LR and increased in HR mice; J-M Heinzmann, S Kloiber, G E Mattos, M Bielohuby, M V Schmidt, R Palme, F Holsboer, M Uhr, M Ising \& C Touma, 2013, unpublished observations), the CORT clearance rates could contribute to the observed differences in HPA axis reactivity and should be therefore considered in future studies. In addition, the expression and possible functional alterations of liver enzymes involved in steroid catabolism (e.g. cytochrome p450 $2 c 39$, hydroxysteroid $17 \beta$ dehydrogenase 9 , and carboxylesterase 1) should also be investigated, as these enzymes were found to be upregulated in a gene expression profiling study with $\mathrm{Cbg}-/-$ mice (Petersen et al. 2006).

Moreover, it is noteworthy that CBG acts as a protein thermocouple (Burton \& Westphal 1972). Studies by Cameron et al. (2010) have shown that the affinity of cortisol for CBG drops approximately by 16 -fold as the temperature increases from 35 to $42^{\circ} \mathrm{C}$, while the binding of cortisol to albumin remains unaffected. Accordingly, a rise in body temperature from 36.5 to $38^{\circ} \mathrm{C}$, as observed in HR, IR, and LR animals subjected to the stress-induced hyperthermia test (Touma et al. 2008), would induce a 50\% reduction in CBG binding affinity for CORT. As a 15-min restraint period would, in theory, induce a similar or even greater change in body temperature, this would result in a decreased CBG binding affinity and consequently proportional CORT release. Thus, we speculate that the difference in CBG levels between the lines could contribute to the increased plasma free CORT after restraint stress by this temperature-dependent process, as the CBG and GC pool is higher in HR than that in IR and LR animals.

Plasma CBG levels in HR, IR, and LR mice already showed significant differences under basal conditions, with the highest abundance of CBG in HR mice, while LR animals presented the lowest amounts (see Fig. 2A).
The first conclusion that can be drawn from this observation is that the significant differences in plasma CBG between the three mouse lines might already affect plasma total and free CORT concentrations under resting conditions. Moreover, in response to stressors such as restraint and forced swimming, the observed differences in plasma total and free CORT levels between the three lines are further enhanced (HR $>I R>L R$, see Fig. $4 A$ and $B$ ). However, plasma CBG levels remained unaffected by the stressors, as well as CBG maximal binding capacity and liver CBG expression (see Figs 1F, 2A and B). Assuming that i) there is no differential storage of CBG per liver cell, even though total CBG storage could be smaller in HR due to a smaller relative liver mass $(\mathrm{HR}<\mathrm{IR}=\mathrm{LR}$, see Supplementary Figure 6 , see section on supplementary data given at the end of this article), ii) there are no differences in CBG half-life in the blood, and iii) CBG mRNA is consistently translated into CBG protein in hepatocytes, we suggest that the observed differences in plasma CBG between the HR, IR, and LR mouse lines are brought about by differences in CBG synthesis rate. Thus, the origin of this differences synthesis levels remains to be determined.

Our findings are not in accord with those published by Qian et al. (2011) who suggested that in rats moderate and strong stressors evoke a fast (within $5 \mathrm{~min}$ ) rise in CBG protein and binding capacity in the blood through a massive release of CBG from the liver. Although we have used a very similar experimental design, we could not observe significant differences in stress-induced CBG protein and binding capacity in the blood of HR, IR, and LR mice. This discrepancy may simply relate to species differences between rats and mice, however, in order to evaluate the clinical relevance of a CBG response to stress, future studies should first address whether the human physiological response to stress is more similar to the pattern observed in rats or in mice.

In order to also consider the other major steroid transport protein, we investigated the role of albumin. Albumin's main functions are the regulation of the osmotic pressure in the blood and the transport of lipophilic compounds including steroids, fatty acids, retinoids, thyroid hormone, and prostaglandins (KraghHansen 1981, 1990, Peters 1985, Baker 2002). Despite its evident role in steroid transport, we did not detect any line differences or stress-induced alterations in albumin protein expression in the plasma or liver of HR, IR, or LR mice (Fig. 3). This might be due to the high abundance of this protein in these two tissues (albumin concentration in the plasma, measured by ELISA $\approx 35 \mathrm{mg} / \mathrm{ml}$, see Supplementary Figure 7 , see section on supplementary

Published by Bioscientifica Ltd. 
data given at the end of this article), implicating that small changes would be difficult to detect in face of the high total amounts.

Regarding plasma total and free CORT (Fig. 1A, B, C and D) and the stress-coping behavior in the FST (Fig. 5), our results are in agreement with the findings reported by Richard et al. (2010). In particular, the neuroendocrine and behavioral endophenotypes of our LR animals, which have lower plasma levels of CBG, clearly parallel those of homozygous CBG knockout mice. LR and $\mathrm{Cbg}-/-$ animals have normal free CORT values at rest but a markedly blunted surge after restraint stress, reflected by the reduced increase in total CORT. Moreover, LR mice and $\mathrm{Cbg}-\mathrm{I}-$ present no changes in anxiety-related behaviors (Touma et al. 2008, Richard et al. 2010) but display a depression-like behavioral phenotype with increased passive stress-coping behavior (floating/ immobility) in the FST (Fig. 5C). Thus, the phenotypic characteristics of LR mice may stem in part from an increased GC clearance due to lower CBG levels.

Previous studies revealed distinct differences between HR, IR, and LR animals not only regarding their CORT secretion but also their sleep architecture, activity rhythms, emotional behavior, cognitive abilities, and neuroendocrine functions as well as neurobiological alterations in the hippocampus and prefrontal cortex resembling several endophenotypes observed in depressed patients, particularly when the melancholic and atypical subtypes of major depression are considered (Touma et al. 2008, 2009, Knapman et al. 2010a,b, 2012). J-M Heinzmann, S Kloiber, G Ebling-Mattos, M Bielohuby, M V Schmidt, R Palme, F Holsboer, M Uhr, M Ising and C Touma (unpublished observations) provided further evidence that the differences in SR between HR, IR, and LR mice are brought about by functional alterations at all levels of the HPA axis (peripheral, central, and regulation). HR mice present enhanced adrenal sensitivity while LR animals show blunted adrenal responsiveness to ACTH. In addition, significantly altered ACTH protein and proopiomelanocortin mRNA expression suggested differential activation of the anterior pituitary. In the brain, acute stress exposure triggered significant differences in neuronal activation of the paraventricular nucleus of the hypothalamus. Therefore, we believe that the differences in plasma $\mathrm{CBG}$ expression levels (HR $>\mathrm{IR}>\mathrm{LR}$ ) reported in this study add another point to the list of molecular changes underlying the behavioral and physiological alterations observed in the SR mouse model.

Taken together, our study has revealed that i) the free CORT increase resembles the pattern of total CORT increase in HR, IR, and LR mice, although with lower concentrations; ii) there is a strong positive correlation between CBG plasma levels and SR, i.e. CORT release, across the three SR mouse lines; iii) although CBG plasma levels, and therefore CBG binding capacity, are higher in HR animals, CORT secretion overshoots the CBG buffering function in response to moderate and strong stressors, resulting in clearly higher free CORT levels in HR compared with IR and LR mice; iv) we did not observe the stress-induced fast CBG increase as described for rats (Qian et al. 2011), indicating a different regulatory mechanism in mice; and v) the lower CBG expression in LR mice could also implicate a faster CORT clearance in these animals and therefore might contribute to the lower CORT levels. However, in HR mice, the high levels of CBG retain a high pool of total CORT in the plasma, at the same time allowing the release of large amounts of free CORT provided by the dynamic equilibrium between CBG-bound and unbound CORT. This process assures an adequate amount of circulating free CORT under basal conditions and a more pronounced effect in response to stressors in HR compared with IR and LR mice.

\section{Supplementary data}

This is linked to the online version of the paper at http://dx.doi.org/10.1530/ JOE-13-0255.

\section{Declaration of interest}

The authors declare that there is no conflict of interest that could be perceived as prejudicing the impartiality of the research reported.

\section{Funding}

This work was supported by the Max Planck Institute of Psychiatry.

\section{Acknowledgements}

The authors like to thank Lisa Tietze for her excellent technical assistance.

\section{References}

Baker ME 2002 Albumin, steroid hormones and the origin of vertebrates. Journal of Endocrinology 175 121-127. (doi:10.1677/joe.0.1750121)

Breuner CW \& Orchinik M 2002 Plasma binding proteins as mediators of corticosteroid action in vertebrates. Journal of Endocrinology $\mathbf{1 7 5}$ 99-112. (doi:10.1677/joe.0.1750099)

Bright GM 1995 Corticosteroid-binding globulin influences kinetic parameters of plasma cortisol transport and clearance. Journal of Clinical Endocrinology and Metabolism 80 770-775. (doi:10.1210/jc.80.3.770)

Burton RM \& Westphal U 1972 Steroid hormone-binding proteins in blood plasma. Metabolism 21 253-276. (doi:10.1016/0026-0495(72)90048-0)

Cameron A, Henley D, Carrell R, Zhou A, Clarke A \& Lightman S 2010

Temperature-responsive release of cortisol from its binding globulin: a

Published by Bioscientifica Ltd. 
protein thermocouple. Journal of Clinical Endocrinology and Metabolism 95 4689-4695. (doi:10.1210/jc.2010-0942)

Cizza G \& Rother KI 2012 Cortisol binding globulin: more than just a carrier? Journal of Clinical Endocrinology and Metabolism 97 77-80. (doi:10.1210/jc.2011-3090)

Droste SK, de Groote L, Atkinson HC, Lightman SL, Reul JM \& Linthorst AC 2008 Corticosterone levels in the brain show a distinct ultradian rhythm but a delayed response to forced swim stress. Endocrinology 149 3244-3253. (doi:10.1210/en.2008-0103)

Droste SK, de Groote L, Lightman SL, Reul JM \& Linthorst AC 2009 The ultradian and circadian rhythms of free corticosterone in the brain are not affected by gender: an in vivo microdialysis study in Wistar rats. Journal of Neuroendocrinology 21 132-140. (doi:10.1111/j.1365-2826. 2008.01811.x)

Fleshner M, Deak T, Spencer RL, Laudenslager ML, Watkins LR \& Maier SF 1995 A long-term increase in basal levels of corticosterone and a decrease in corticosteroid-binding globulin after acute stressor exposure. Endocrinology 136 5336-5342. (doi:10.1210/en.136.12.5336)

Hammond GL \& Lahteenmaki PL 1983 A versatile method for the determination of serum cortisol binding globulin and sex hormone binding globulin binding capacities. Clinica Chimica Acta 132 101-110. (doi:10.1016/0009-8981(83)90237-1)

Hammond GL, Smith CL, Goping IS, Underhill DA, Harley MJ, Reventos J, Musto NA, Gunsalus GL \& Bardin CW 1987 Primary structure of human corticosteroid binding globulin, deduced from hepatic and pulmonary cDNAs, exhibits homology with serine protease inhibitors. PNAS $\mathbf{8 4}$ 5153-5157. (doi:10.1073/pnas.84.15.5153)

Heinzmann JM, Thoeringer CK, Knapman A, Palme R, Holsboer F, Uhr M, Landgraf R \& Touma C 2010 Intrahippocampal corticosterone response in mice selectively bred for extremes in stress reactivity: a microdialysis study. Journal of Neuroendocrinology 22 1187-1197. (doi:10.1111/j.13652826.2010.02062.x)

Henley DE \& Lightman SL 2011 New insights into corticosteroid-binding globulin and glucocorticoid delivery. Neuroscience 180 1-8. (doi:10.1016/j.neuroscience.2011.02.053)

de Kloet ER, Voorhuis TA, Leunissen JL \& Koch B 1984 Intracellular CBG-like molecules in the rat pituitary. Journal of Steroid Biochemistry 20 367-371. (doi:10.1016/0022-4731(84)90235-8)

Knapman A, Heinzmann JM, Hellweg R, Holsboer F, Landgraf R \& Touma C $2010 a$ Increased stress reactivity is associated with cognitive deficits and decreased hippocampal brain-derived neurotrophic factor in a mouse model of affective disorders. Journal of Psychiatric Research $\mathbf{4 4}$ 566-575. (doi:10.1016/j.jpsychires.2009.11.014)

Knapman A, Heinzmann JM, Holsboer F, Landgraf R \& Touma C $2010 b$ Modeling psychotic and cognitive symptoms of affective disorders: disrupted latent inhibition and reversal learning deficits in highly stress reactive mice. Neurobiology of Learning and Memory 94 145-152. (doi:10.1016/j.nlm.2010.04.010)

Knapman A, Kaltwasser SF, Martins-de-Souza D, Holsboer F, Landgraf R, Turck CW, Czisch M \& Touma C 2012 Increased stress reactivity is associated with reduced hippocampal activity and neuronal integrity along with changes in energy metabolism. European Journal of Neuroscience 35 412-422. (doi:10.1111/j.1460-9568.2011.07968.x)

Kragh-Hansen U 1981 Molecular aspects of ligand binding to serum albumin. Pharmacological Reviews 33 17-53.

Kragh-Hansen U 1990 Structure and ligand binding properties of human serum albumin. Danish Medical Bulletin 37 57-84.

Kuhn RW, Green AL, Raymoure WJ \& Siiteri PK 1986 Immunocytochemical localization of corticosteroid-binding globulin in rat tissues. Journal of Endocrinology 108 31-36. (doi:10.1677/joe.0.1080031)

Lewis JG, Bagley CJ, Elder PA, Bachmann AW \& Torpy DJ 2005 Plasma free cortisol fraction reflects levels of functioning corticosteroid-binding globulin. Clinica Chimica Acta 359 189-194. (doi:10.1677/joe.0.1080031)

Mendel CM 1989 The free hormone hypothesis: a physiologically based mathematical model. Endocrine Reviews 10 232-274. (doi:10.1210/ edrv-10-3-232)
Minni AM, Dorey R, Pierard C, Dominguez G, Helbling JC, Foury A, Beracochea D \& Moisan MP 2012 Critical role of plasma corticosteroidbinding-globulin during stress to promote glucocorticoid delivery to the brain: impact on memory retrieval. Endocrinology 153 4766-4774. (doi:10.1210/edrv-10-3-232)

Moisan MP 2010 Genotype-phenotype associations in understanding the role of corticosteroid-binding globulin in health and disease animal models. Molecular and Cellular Endocrinology 316 35-41. (doi:10.1016/ j.mce.2009.07.017)

Moisan MP 2013 CBG: a cortisol reservoir rather than a transporter. Nature Reviews. Endocrinology 9 78. (doi:10.1038/nrendo.2012.134-c1)

Mopert B, Herbert Z, Caldwell JD \& Jirikowski GF 2006 Expression of corticosterone-binding globulin in the rat hypothalamus. Hormone and Metabolic Research 38 246-252. (doi:10.1055/s-2006-925344)

Nakhla AM, Leonard J, Hryb DJ \& Rosner W 1999 Sex hormone-binding globulin receptor signal transduction proceeds via a G protein. Steroids 64 213-216. (doi:10.1016/S0039-128X(98)00084-1)

Neufeld JH, Breen L \& Hauger R 1994 Extreme posture elevates corticosterone in a forced ambulation model of chronic stress in rats. Pharmacology, Biochemistry, and Behavior 47 233-240. (doi:10.1016/ 0091-3057(94)90005-1)

Perogamvros I, Ray DW \& Trainer PJ 2012 Regulation of cortisol bioavailability-effects on hormone measurement and action. Nature Reviews. Endocrinology 8 717-727. (doi:10.1038/nrendo.2012.134)

Peters T Jr 1985 Serum albumin. Advances in Protein Chemistry 37 161-245.

Petersen HH, Andreassen TK, Breiderhoff T, Brasen JH, Schulz H, Gross V, Grone HJ, Nykjaer A \& Willnow TE 2006 Hyporesponsiveness to glucocorticoids in mice genetically deficient for the corticosteroid binding globulin. Molecular and Cellular Biology 26 7236-7245. (doi:10.1128/MCB.00400-06)

Qian X, Droste SK, Gutierrez-Mecinas M, Collins A, Kersante F, Reul JM \& Linthorst AC 2011 A rapid release of corticosteroid-binding globulin from the liver restrains the glucocorticoid hormone response to acute stress. Endocrinology 152 3738-3748. (doi:10.1210/en.2011-1008)

Rice WR 1989 Analyzing tables of statistical tests. Evolution 43 223-225.

Richard EM, Helbling JC, Tridon C, Desmedt A, Minni AM, Cador M, Pourtau L, Konsman JP, Mormede P \& Moisan MP 2010 Plasma transcortin influences endocrine and behavioral stress responses in mice. Endocrinology 151 649-659. (doi:10.1210/en.2009-0862)

Rothschild MA, Oratz M \& Schreiber SS 1972 Albumin metabolism. Progress in Liver Diseases 4 19-29.

Sandberg AA, Woodruff M, Rosenthal H, Nienhouse S \& Slaunwhite WR Jr 1964 Transcortin: a corticosteroid-binding protein of plasma. Vii. Half-life in normal and estrogen-treated subjects. Journal of Clinical Investigation 43 461-466. (doi:10.1172/JCI104931)

Siegel S \& Castellan NJ 1988 Nonparametric Statistics for the Behavioral Sciences, Edn 2. New York: McGraw-Hill Book Company.

Siiteri PK, Murai JT, Hammond GL, Nisker JA, Raymoure WJ \& Kuhn RW 1982 The serum transport of steroid hormones. Recent Progress in Hormone Research 38 457-510.

Sivukhina E, Helbling JC, Minni AM, Schafer HH, Pallet V, Jirikowski GF \& Moisan MP 2012 Intrinsic expression of transcortin in neural cells of the mouse brain: a histochemical and molecular study. Journal of Experimental Biology 216 245-252. (doi:10.1242/jeb.076893)

Slaunwhite WR Jr \& Sandberg AA 1959 Transcortin: a corticosteroidbinding protein of plasma. Journal of Clinical Investigation 38 384-391. (doi:10.1172/JCI103812)

Spencer RL, Miller AH, Moday H, McEwen BS, Blanchard RJ, Blanchard DC \& Sakai RR 1996 Chronic social stress produces reductions in available splenic type II corticosteroid receptor binding and plasma corticosteroid binding globulin levels. Psychoneuroendocrinology 21 95-109. (doi:10.1016/0306-4530(95)00020-8)

Thoeringer CK, Sillaber I, Roedel A, Erhardt A, Mueller MB, Ohl F, Holsboer F \& Keck ME 2007 The temporal dynamics of intrahippocampal corticosterone in response to stress-related stimuli with different emotional and physical load: an in vivo microdialysis study in 
C57BL/6 and DBA/2 inbred mice. Psychoneuroendocrinology 32 746-757. (doi:10.1016/j.psyneuen.2007.05.005)

Touma C, Bunck M, Glasl L, Nussbaumer M, Palme R, Stein H, Wolferstatter M, Zeh R, Zimbelmann M, Holsboer F et al. 2008 Mice selected for high versus low stress reactivity: a new animal model for affective disorders. Psychoneuroendocrinology 33 839-862. (doi:10.1016/j.psyneuen.2008.03.013)

Touma C, Fenzl T, Ruschel J, Palme R, Holsboer F, Kimura M \& Landgraf R 2009 Rhythmicity in mice selected for extremes in stress reactivity: behavioural, endocrine and sleep changes resembling endophenotypes of major depression. PLOS ONE $\mathbf{4}$ e4325. (doi:10.1371/journal.pone. 0004325)

Tronche C, Pierard C, Coutan M, Chauveau F, Liscia P \& Beracochea D 2010 Increased stress-induced intra-hippocampus corticosterone rise associated with memory impairments in middle-aged mice. Neurobiology of Learning and Memory 93 343-351. (doi:10.1016/j.nlm.2009.11.009)

Weiser JN, Do YS \& Feldman D 1979 Synthesis and secretion of corticosteroid-binding globulin by rat liver. A source of heterogeneity of hepatic corticosteroid-binders. Journal of Clinical Investigation $\mathbf{6 3}$ 461-467. (doi:10.1172/JCI109323)

Received in final form 12 September 2013

Accepted 17 September 2013

Accepted Preprint published online 17 September 2013
Published by Bioscientifica Ltd. 